Sādhanā Vol. 36, Part 4, August 2011, pp. 489-504. (C) Indian Academy of Sciences

\title{
An inverse heat transfer problem for optimization of the thermal process in machining
}

\author{
M GOSTIMIROVIC*，P KOVAC and M SEKULIC \\ Department of Production Engineering, Faculty of Technical Science, \\ University of Novi Sad, Trg D. Obradovica 6, 21000 Novi Sad, Serbia \\ e-mail: maring@uns.ac.rs; pkovac@uns.ac.rs; milenkos@uns.ac.rs
}

MS received 31 March 2010; revised 11 February 2011; accepted 27 May 2011

\begin{abstract}
It is evident that machining process causes development of large quantities of thermal energy within a relatively narrow area of the cutting zone. The generated thermal energy and the problems of its evacuation from the cutting zone account for high temperatures in machining. These increased temperatures exert a pronounced negative effect on the tool and workpiece. This paper takes a different approach towards identification of the thermal process in machining, using inverse heat transfer problem. Inverse heat transfer method allows the closest possible experimental and analytical approximation of thermal state for a machining process. Based on a temperature measured at any point within a workpiece, inverse method allows determination of a complete temperature field in the cutting zone as well as the heat flux distribution on the tool/workpiece interface. By knowing the heat flux function, one defines criterium and method of optimization, the inverse heat transfer problem transforms into extreme case. Now, the task of optimization is to determine most favourable ratio between heat flux parameters in order to preserve exploitation properties of the tool and workpiece.
\end{abstract}

Keywords. Machining process; thermal energy; temperature; heat flux; inverse problem; optimization.

\section{Introduction}

Modern manufacturing is facing complex demands on a daily basis. System flexibility, productivity of manufacturing and high levels of machining quality and accuracy, are the most vital demands facing the market-oriented industrial systems. Only modern equipped industrial systems shall be able to adjust their manufacturing process to these high market demands.

There can be little doubt that machining technologies shall remain important in modern manufacturing industry. Especially important are material machining methods as the integral part of the technological process of product manufacturing and assembly. Basic advantages of machining process are high productivity, precision and surface quality with the ability to cope with hard

*For correspondence 
materials and complex surfaces (Khachfe \& Jarny 2000). However, due to process of chip formation during machining, it is evident that machining methods cause the development of large quantities of thermal energy within the cutting zone (Stephenson 1991).

The generated thermal energy, located within a relatively narrow area of the cutting zone, causes high temperatures (Milikic \& Gostimirovic 1994). These increased temperatures instantaneously burst to a maximum, causing various physical and chemical changes in the cutting zone and exerting a pronounced negative effect on the tool and workpiece. Since the main task of machining is to achieve satisfactory part quality with as large productivity as possible, special attention is focused on the effect that the cutting temperatures have on the change of material properties in the workpiece surface layer and machining accuracy (Shaw 2003). If the temperatures thus generated are high enough to cause structural and phase transformations of the workpiece material, the machined surface shall suffer from a number of disadvantages. Should, in addition, dimensional errors appear as well, the overall effect can substantially diminish exploitation features of the finished part. Obviously, efficient machining of parts, free of thermal defects in the workpiece, requires methods for optimization of thermal phenomena by regulation of cutting temperatures.

Systematic research in machining technologies has yielded a number of various analytical and experimental methods for determination of temperatures not only in the narrow and wider cutting zone, but also in the machining system (Gostimirovic \& Kovac 2008). Due to rudimentary measuring equipment, first research on temperatures in machining was mostly theoretical. Later advancements in measuring equipment allowed development of various experimental methods for temperature measurement in machining, which have been undergoing modification and improvement until today. However, although the temperature is an essential parameter in machining, its utilization for the purpose of optimization of machining is fairly complex. The main obstacle on the road to its utilization for optimization purposes lies in the difficult monitoring of cutting temperatures. Therefore, efforts are aimed at improving the existing and development of novel measurement methods, while at the same time focusing on analytical optimization models which can successfully relate to cutting temperatures (Lin 1995).

As the research so far has shown, non-stationary and non-linear processes that involve intensive heat conduction, such as machining, can be successfully solved using inverse problems of heat transfer (Özisik \& Orlande 2000; Shidfor \& Tawakoli 2002; Tikhe \& Deshmukh 2005). The inverse problems are today successfully applied in identification, design, control and optimization of thermal processes and plants. In the case of machining, the inverse problems so far has mostly been used to identify processes by approximating heat flux or temperature field in the cutting zone (Kim et al 2006).

When the inverse heat transfer problem is transformed into an extreme case it is practically the only way to optimize heat loading on the workpiece (Alifanov 1994). For a known temperature measured at a point within the workpiece, numerical methods are used to approximate the total temperature field as well as the heat flux in the cutting zone. For the selected model of optimization, quality criteria and boundaries, it is possible to arrive at optimal machining conditions by controlling the heat flux.

\section{Inverse heat transfer problem of machining}

\subsection{General case of inverse problem}

The process of heat transfer between solid bodies or between a system and its environment is mostly considered from the standpoint of mutual relations between input and output process parameters. It is widely accepted that such process can be schematized as in figure 1. 


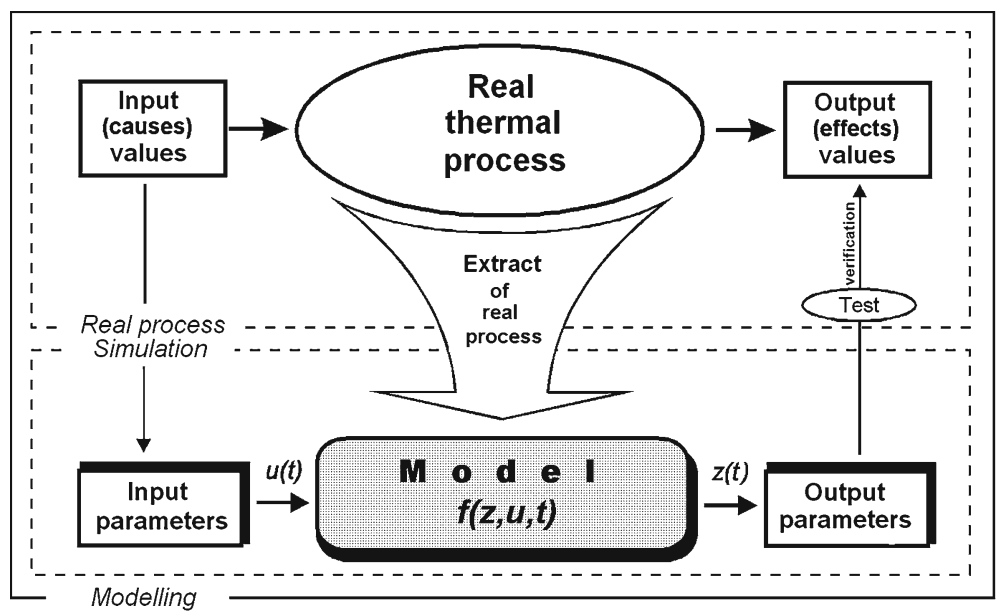

Figure 1. Diagram of a thermal process.

The first step in the research of any thermal phenomenon is to model the real process. This means that development of a model which is valid over a narrow domain limited by boundary conditions. The model, which describes a segment of the real process, correlates input $\boldsymbol{u}(t)$ and output $z(t)$ parameters which define the state of the process at every moment in time $t$.

If the input parameters $\boldsymbol{u}(t)$ are known and output parameters $\boldsymbol{z}(t)$ define process state in time, then the output parameters are a function of input parameters, i.e.;

$$
z=f(u, t) .
$$

The real thermal process is most often described analytically. The goal is to set up a most adequate analytical model, while, on the other side, keeping its form as simple as possible in order to facilitate solution. Given the right mathematical method, the model thus defined, solves problems quickly and efficiently. Analytical model of thermal process most often takes the form of a system of differential and algebraic equations. Since such form is easily transformed into algorithm and efficiently processed on computer, the differential models are widespread today in the investigation of thermal processes.

If for the adopted thermal model there exist unique conditions (boundary and initial conditions, thermal and physical properties and geometry of a body or a system), then any particular input parameters of the thermal process shall result in that same or any other thermal state defined by the temperature field of the analysed object. Determination of the input-output relationship is the direct heat transfer problem. Conversely, the inverse heat transfer problem is used to find input characteristics of the process for the known temperature field (Beck et al 1985; Andreas 1989).

If for every unknown parameter $\boldsymbol{u}$ there is a linear, smooth operator $\mathbf{A}$ which allows determination of output parameter $z$, the general case of inverse problem is formulated by the following equation:

$$
\mathbf{A} u=z .
$$

If we represent the unknown input parameter of the thermal state with $\boldsymbol{u}(t)$, and if $\boldsymbol{z}(t)$ denotes the known output parameter of the process, in area of $\boldsymbol{D}$, then the inverse problem becomes:

$$
\frac{d z}{d t}=f(z, u, t)
$$


with initial, additional and boundary conditions:

$$
\begin{gathered}
\left.z\right|_{t=0}=z_{0} \\
\left.z\right|_{D=K}=z_{K} \\
\left.z^{\prime}\right|_{D=S}=u_{S} .
\end{gathered}
$$

In equation (3), $\boldsymbol{u}_{S}$ is the solution, i.e., the surface heat flux and temperature on the boundary of body $S$, while function $z_{K}$ represents the temperatures measured outside the body at a point $\boldsymbol{K}$.

\subsection{Modelling of thermal state in machining}

One of the essential problems of machining is the knowledge of fundamental laws which regulate generation and distribution of thermal energy as well as the character of the temperature fields in the cutting zone. The quantity of thermal energy generated in the machining process is equivalent to mechanical work applied to machining. Conversion of mechanical energy into thermal energy takes place within several characteristic zones, with some zones partially overlapping, figure 2 (left). On the flank and face surface of a tool, mechanical work is transformed into heat due to intensive friction between the tool, machined surface and chip. Further transformation of mechanical work into heat takes place during chip formation, through plastic deformation of workpiece material. The thermal energy thus generated is evacuated by conduction, convection and radiation. Heat sinks, which take away the thermal energy from the cutting zone, are tool, workpiece, chip, coolant and environment.

Characteristics of heat sources and sinks depend on a large number of influential factors which contribute to a process of chip formation during machining. Therefore, it is very difficult to define the character of thermal energy in the cutting zone. Similar to other thermal processes, correct evaluation of the character of thermal energy in machining requires knowledge of the following: type, dimension, shape, distribution law, velocity, time and strength of thermal energy.

The role of mathematical theory behind thermal phenomena in machining is to adopt the most adequate model of workpiece, tool and their inter-relationships. If the numerous variable parameters were taken into consideration, analytical modelling of the machining process would become an impossible task. Therefore, some simplifications are necessary where the final solution is verified by experiments. Despite simplification, such analytical and experimental model yields reliable results.

One can assume that the total heat source on the machining process $\boldsymbol{q}$ is the result of friction between the tool, workpiece and chip in the workpiece material shear plane. This total heat
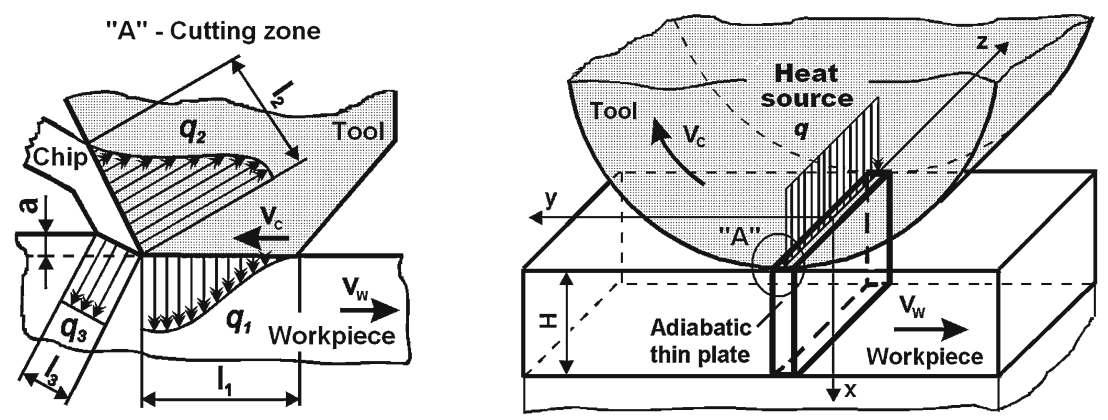

Figure 2. Model of a thermal state in machining process. 
source, whose strength varies within a narrow range, acts continuously, shifting across the workpiece surface with constant velocity. The shape and dimensions of the heat source depend on the tool/workpiece interface. In machining, considering that the cutting depth is many times smaller than the length and width of tool/workpiece interface, the heat source can be treated as a strip of infinite length and constant heat distribution, figure 2 (right). The assumption of constant heat distribution across the interface is a valid approximation in case of the heating of thin surface layers of tool and workpiece material.

\subsection{Inverse problem in machining}

In the previously defined thermal model of machining, heat transfer alongside tool/workpiece interface can be disregarded, thus rendering the workpiece surface isolated. Furthermore, if we disregard the dissipation of heat flow in the direction of heat source movement, then the workpiece can be approximated with a semi-infinite plate, figure 3. Substitution of the real workpiece with series of adiabatic thin plates is completely justified, bearing in mind that the heat source in machining is generated within a small volume of workpiece material while the heat loading of the surface workpiece layer is considered depth-wise.

In that case, the following is a more general case of differential equation of a one-dimensional heat conduction:

$$
C(T(x, t)) \frac{\partial T(x, t)}{\partial t}=\frac{\partial}{\partial x}\left(k(T(x, t)) \frac{\partial T(x, t)}{\partial x}\right) \quad \begin{aligned}
& x \in(0, H) \\
& t \in\left(0, t_{m}\right]
\end{aligned},
$$

where $\boldsymbol{T}=T(x, t)$ - workpiece temperature at point coordinate $\boldsymbol{x}$ at moment $\boldsymbol{t} ; \boldsymbol{k}$ - thermal conductivity; $\boldsymbol{C}=\rho c$ - specific heat capacity ( $\rho$ - material density, $c$ - specific heat); $\boldsymbol{H}$ thickness of the surface layer of workpiece material and $\boldsymbol{t}_{m}$ - largest time increment.

Now the analytical form of inverse heat transfer problem for machining can be described with differential equation (4) should be considered in conjunction with the initial temperature distribution, additional and boundary conditions.

The initial condition refers to defining a temperature profile in the workpiece at the initial moment $\boldsymbol{t}=0$ :

$$
\left.T(x, t)\right|_{t=0}=T_{0}(x) \quad x \in[0, H] .
$$

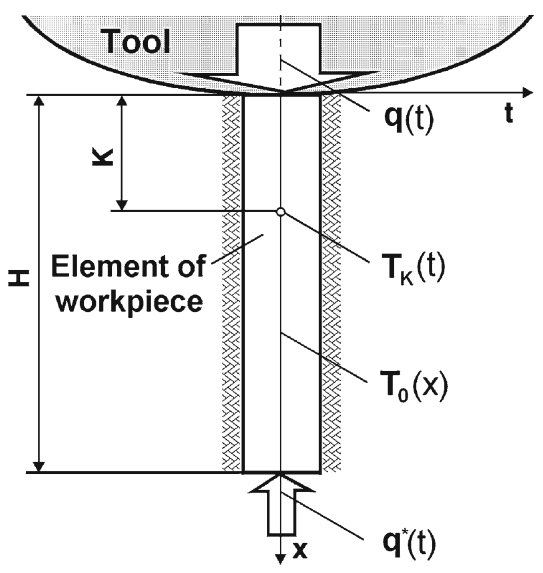

\section{Initial condition}

$\boldsymbol{T}_{0}(x)$ - initial temperature

Additional condition

$\boldsymbol{T}_{K}(t)$ - known temperature

Boundary condition

$\boldsymbol{q}(t)$ - unknown heat flux

$q^{*}(t)$ - known heat flux

Figure 3. Schema of a uni-dimensional inverse problem of the machining process. 
An additional condition is the fact that at the point of workpiece $x=K(0<K \leq H)$, there is a known temperature, measured during a time interval:

$$
\left.T(x, t)\right|_{x=K}=T_{K}(t) \quad t \in\left[0, t_{m}\right] .
$$

Boundary conditions for the considered workpiece surface layer are defined by the boundary condition of the second order. The lower boundary condition is defined by the known heat flux:

$$
-\left.k(T(x, t)) \frac{\partial T(x, t)}{\partial x}\right|_{x=H}=q^{*}(t) \quad t \in\left[0, t_{m}\right],
$$

while the upper boundary condition is defined by the unknown heat flux over the tool/workpiece interface.

$$
-\left.k(T(x, t)) \frac{\partial T(x, t)}{\partial x}\right|_{x=0}=q(t) \quad t \in\left[0, t_{m}\right] .
$$

The final solution of the inverse heat transfer problem is the heat flux on tool/workpiece interface $\boldsymbol{q}=q(t)$, and the temperature field $\boldsymbol{T}=T(x, t)$ throughout the entire elementary part of workpiece, $\boldsymbol{D}=\left\{(x, t): x \in[0, H], t \in\left[0, t_{m}\right]\right\}$.

Furthermore, we can transform inverse heat transfer problem into an extreme case using some method of optimization. Given the input parameters, this would allow us to determine the optimal thermal state of the process for the analytical model in hand, so as to satisfy the state and boundary functions for the given optimization criterion.

For machining, optimization of the thermal process is to determine the function of the process state $\boldsymbol{T}=T(x, t)$, and the control function $\boldsymbol{q}=q(t)$, so as to satisfy the analytical form of the inverse problem for the machining process, as defined by equations (4) to (8). These functions are determined under the condition that the known temperature at a particular fixed point $\boldsymbol{T}_{K}=$ $T(K, t)$ is consistent with the temperature $\boldsymbol{T}_{q}=\boldsymbol{T}(q, K, t)$ which is calculated based on control function $\boldsymbol{q}(t)$ :

$$
J(q)=\int_{0}^{t_{m}}\left[T_{q}(q, K, t)-T_{K}(K, t)\right]^{2} d t+r_{c} \cdot \Omega(q),
$$

where $\boldsymbol{r}_{c}$ - weight coefficient factor and $\boldsymbol{\Omega}(q)$ - stabilizing functional.

It is known with certainty that the addition of the stabilizing functional contributes to the quality of control system. However, the influence of stabilizer $\boldsymbol{\Omega}(q)$ is quite low and can be disregarded, especially if the number of included parameters is small.

\section{Numerical solution of inverse heat transfer problem}

\subsection{Implicit method of finite differences}

Due to high complexity, differential equations of the second order which describe the process of heat conduction in machining are mostly solved using numerical methods. These methods transform exact differential equations into approximate algebraic equations.

The first step with every numerical method is the discretization of space, i.e., approximation of a thin, isolated plate of workpiece by a number of elementary pieces $\Delta \boldsymbol{x}$, figure 4 . When 
$\left.D_{1}=\{(x, t): x \in[0, K\}], t \in\left[0, t_{m}\right]\right\} \quad D_{2}=\left\{(x, t): x \in[K, H], t \in\left[0, t_{m}\right]\right\}$

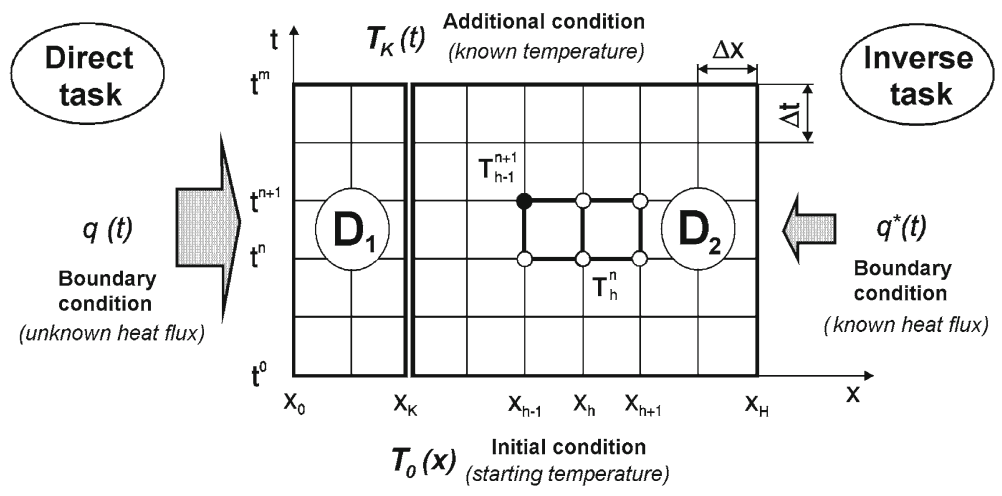

Figure 4. The shape of a uni-dimensional mesh of inverse heat transfer problem.

dealing with a non-stationary heat conduction problem, the time of temperature change and heat flux are discretized by a $\Delta t$ increment.

To solve the partial differential equation (4) an implicit form of the finite differences method was chosen. The concept of this method very much resembles the physical process, where the temperature or heat flux at each observed point is calculated after a time increment as the result of heat exchange with the neighbouring points. Based on the five known temperatures at the neighbouring points, the temperature at the next moment in time is calculated.

The results obtained by inverse problem of the heavily loaded thermal processes, are highly prone to errors due to change of thermal and physical material properties with temperature. Therefore, solving of a non-linear inverse problem requires the thermal and physical material properties to be expressed in the form of approximate functions of temperature, bearing in mind the conditions of smooth differentiation.

In that case, the analytical model given in (4) should be substituted by another, more appropriate form:

$$
\frac{1}{\alpha(T)} \frac{\partial T}{\partial t}=\frac{\partial^{2} T}{\partial x^{2}}+\frac{k^{\prime}(T)}{k(T)}\left(\frac{\partial T}{\partial x}\right)^{2} \quad \begin{aligned}
x & \in(0, H) \\
t & \in\left(0, t_{m}\right]
\end{aligned}
$$

where $\boldsymbol{\alpha}=k / C$ - thermal diffusivity and $k^{\prime} \equiv d k / d T$.

Introducing an even-spaced triangular mesh $\left\{\boldsymbol{x}_{h}=h \cdot \Delta x, \boldsymbol{h}=0, H ; \boldsymbol{t}_{n}=n \cdot \Delta t, \boldsymbol{n}=\right.$ $0, m\}$ and using Taylor approximation, the first and second derivatives of the partial differential equation (10) can be written in the form of finite differences (Kreith \& Black 1980):

$$
\begin{aligned}
& \left.\frac{\partial^{2} T}{\partial x^{2}}\right|_{h} ^{n}=\frac{T_{h+1}^{n+1}-2 T_{h}^{n+1}+T_{h-1}^{n+1}}{\Delta x^{2}}+\varepsilon_{A}\left(\Delta x^{2}\right) \\
& \left.\frac{\partial T}{\partial x}\right|_{h} ^{n}=\frac{T_{h+1}^{n}-T_{h-1}^{n}}{2 \Delta x}+\varepsilon_{A}\left(\Delta x^{2}\right) \\
& \left.\frac{\partial T}{\partial t}\right|_{h} ^{n}=\frac{T_{h}^{n+1}-T_{h}^{n}}{\Delta t}+\varepsilon_{A}(\Delta t),
\end{aligned}
$$

where $\boldsymbol{\varepsilon}_{A}$ is an estimation error. 
Substituting equations (11) into equation (10) one derives equation of non-stationary unidimensional heat conduction in the form of finite differences:

$$
-r_{h}^{n} T_{h-1}^{n+1}+\left(1+2 r_{h}^{n}\right) T_{h}^{n+1}-r_{h}^{n} T_{h+1}^{n+1}=T_{h}^{n}+r_{h}^{n} \frac{k_{h}^{\prime n}}{4 k_{h}^{n}}\left(T_{h+1}^{n}-T_{h-1}^{n}\right)^{2},
$$

where $r_{h}^{n}=\alpha_{h}^{n} \Delta t / \Delta x^{2}$.

Discrete representation of heat flux $\boldsymbol{q}(t)$ and $\boldsymbol{q}^{*}(t)$ requires introduction of finite differences of the second order, bearing in mind the accumulation of heat within a particular element of $\Delta \boldsymbol{x}$ thickness (Alifanov 1994):

$$
\begin{aligned}
q^{n+1} & =k_{0}^{n} \frac{T_{0}^{n+1}-T_{1}^{n+1}}{\Delta x}+C_{0}^{n} \frac{\Delta x}{2} \frac{T_{0}^{n+1}-T_{0}^{n}}{\Delta t} \\
q^{* n+1} & =k_{H}^{n} \frac{T_{H}^{n+1}-T_{H-1}^{n+1}}{\Delta x}+C_{H}^{n} \frac{\Delta x}{2} \frac{T_{H}^{n+1}-T_{H}^{n}}{\Delta t} .
\end{aligned}
$$

The discrete form of the initial temperature distribution is:

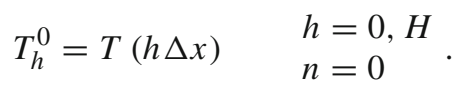

Expressions (12) to (14) result in a system of linear algebraic equations in implicit form of finite differences. The equations are used to calculate the unknown heat flux $\boldsymbol{q}^{n+1}$ and the temperature field in the cutting zone $\boldsymbol{T}_{h}^{n+1}(h=0,1, \ldots, K-1, K+1, \ldots, H)$ as follows:

$$
[\mathbf{R}] \cdot\{\mathbf{T}\}=\{\mathbf{B}\} .
$$

Solving the matrix system (15) requires the initial task to be divided into two parts.

First, a standalone system is calculated:

$$
\left[\mathbf{R}_{\mathbf{2}}\right] \cdot\left\{\mathbf{T}_{\mathbf{2}}\right\}=\left\{\mathbf{B}_{\mathbf{2}}\right\}
$$

that is:

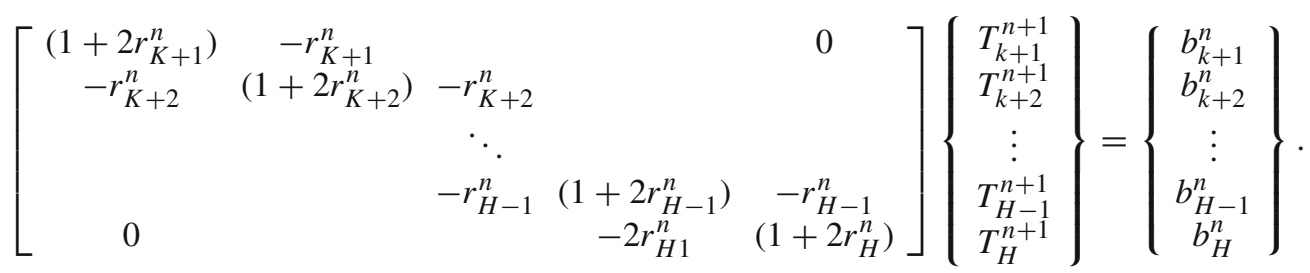

The matrix equation (16) is solved as the direct task of heat conduction within the area $\boldsymbol{D}_{2}=$ $\left\{(x, t): x \in[K, H], t \in\left[0, t_{m}\right]\right\}$. The solution yields the unknown temperatures $\boldsymbol{T}_{h}^{n+1}(h=$ $K+1, \ldots, H)$.

Once vector $\mathbf{T}_{2}$ is determined, one can tackle the problem of inverse heat transfer in the area of $\boldsymbol{D}_{1}=\left\{(x, t): x \in[0, K], t \in\left[0, t_{m}\right]\right\}$ using the system:

$$
\left[\mathbf{R}_{1}\right] \cdot\left\{\mathbf{T}_{1}\right\}=\left\{\mathbf{B}_{1}\right\}
$$


that is:

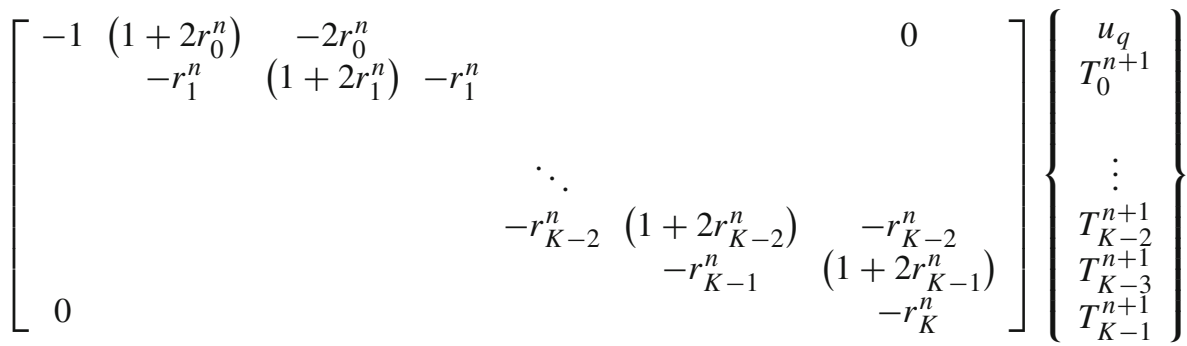

$$
\begin{aligned}
& =\left\{\begin{array}{c}
0 \\
b_{1}^{n} \\
\vdots \\
b_{K-1}^{n} \\
b_{K-2}^{n} \\
\hat{b}_{K}^{n}
\end{array}\right\},
\end{aligned}
$$

in equations (16) and (17) where:

$$
\begin{array}{ll}
u_{q}=2 \frac{\alpha_{0}^{n} \Delta t}{k_{0}^{n} \Delta x} q^{n+1}+T_{0}^{n} & \\
b_{\left.i\right|_{i=k+i=2 ; i-1} ^{i=1 ; k-2}=b_{i}^{n}} & b_{i}^{n}=T_{i}^{n}+r_{i}^{n} \frac{k_{i}^{\prime n}}{4 k_{i}^{n}}\left(T_{i+1}^{n}-T_{i-1}^{n}\right)^{2} \\
b_{\substack{i=k-1 \\
i=k+1}}=b_{i}^{n}+r_{i}^{n} T_{k}^{n+1} & \\
b_{H}=T_{H}^{n}+2 \frac{a_{H}^{n} \Delta t}{k_{H}^{n} \Delta x} q^{* n+1} & \hat{b}_{K}^{n}=b_{K}^{n}-\left(1+2 r_{K}^{n}\right) T_{K+1}^{n+1}+r_{K}^{n} \cdot T_{K+1}^{n+1} .
\end{array}
$$

From the system (17), starting from the first unknown temperature $T_{K-1}^{n+1}$, vector $\mathbf{T}_{\mathbf{1}}$ is calculated. This vector represents the unknown heat flux $\boldsymbol{q}^{n+1}$ and the unknown temperature $\boldsymbol{T}_{h}^{n+1}(h=$ $0, \ldots, K-1)$.

\subsection{Iterative optimization method}

In order to establish optimal control over the previously defined extreme case of inverse problem, the total search iterative method was selected as the method of optimization. It allows optimization of linear and nonlinear goal functions, with or without constraints. It is efficient and allows relatively simple solution of even the most complex optimization problem.

Using iterative algorithms, through gradual approach to the maximum over a number of successive steps, the smooth function $\boldsymbol{q}(t)$ is given in the form of a finite vector:

$$
\mathbf{q}=\left\{q^{1}, q^{2}, \ldots, q^{m}\right\}^{\mathrm{T}}
$$


where $\boldsymbol{q}^{n}=q\left(t_{n}\right)$ for $\boldsymbol{n}=1, m$ are components derived by discretization of the chosen time mesh.

For computer application, the integral quality criterion, equation (9), is replaced by the finite sum:

$$
J(\mathbf{q}) \cong \Delta t \sum_{n=1}^{m}\left[T_{q}\left(\mathbf{q}, K, t_{n}\right)-T_{K}\left(K, t_{n}\right)\right]^{2},
$$

where $\Delta \boldsymbol{t}$ - time increment and $\boldsymbol{t}_{n}$ - time point of an even discretization of smooth functions $\boldsymbol{T}_{q}\left(\mathbf{q}, K, t_{n}\right)$ and $\boldsymbol{T}_{K}\left(K, t_{n}\right)$.

In order to minimize the differences between the known and the calculated temperatures, an iterative method of optimization is used, which gives exact solution using the following form:

$$
\mathbf{q}_{i+1}(t)=\mathbf{q}_{i}(t)+\Delta \mathbf{q}_{i}(t) \quad i=0,1,2, \ldots,
$$

where $\mathbf{q}_{0}(t)$ - initial approximation and $\Delta \mathbf{q}_{i}(t)$ - iteration step.

$J\left(\mathbf{q}_{i}\right)$ is calculated by applying the iterative optimization algorithm for particular values of the functional $\mathbf{q}_{i}(t)$. The procedure is repeated, decreasing the value of the functional $\boldsymbol{J}\left(\mathbf{q}_{i+1}\right)<$ $J\left(\mathbf{q}_{i}\right)$, until its minimum is reached. The iterative gradient method procedure is considered finished for a sufficiently small functional, which means that the calculated $\boldsymbol{T}_{q}\left(\mathbf{q}, K, t_{n}\right)$ and the measured temepartures $\boldsymbol{T}_{K}\left(K, t_{n}\right)$ are very close or almost identical.

Our consideration of the problem of optimization using iterative method supposes that it is the unknown solution of the inverse problem that is minimizing the goal function. However, if the input data contain certain error and discretization intervals are small enough to preclude self-regulation, the final solution shall be approached in an oscillating manner. It is therefore advisable to stop the iterative process at some iteration in order to avoid substantial oscillations of the solution.

Accordingly, for the allowed level of optimization (equation (20)), the following stopping criterion is valid:

$$
J(\mathbf{q}) \cong \varepsilon^{2} .
$$

Here $\varepsilon$ represents estimation of input data error, and is calculated as:

$$
\varepsilon^{2}=\int_{0}^{t_{m}} \sigma^{2}(t) d t \cong \Delta t \sum_{n=1}^{m}\left[T_{K}\left(K, t_{n}\right)-T_{m}\right],
$$

where $\sigma^{2}(t)$ - dispersion of function $\boldsymbol{T}_{K}\left(K, t_{n}\right)$ and $\boldsymbol{T}_{m}=\Sigma \boldsymbol{T}_{K}\left(K, t_{n}\right) / m$ - average value of measured temperatures.

\section{Verification of inverse problem in the machining}

\subsection{Experimentation}

As the proposed system uses experiment and analytical model to optimize thermal process in machining, it requires distribution of temperatures to be determined experimentally at a point within the workpiece, figure 5(bottom). Temperatures in the workpiece were measured at various distances from the measuring point to the contact surface of the workpiece and the tool.

The experimental work was carried out on a surface grinding machine ( $\gg$ Majevica $\ll$ type $\mathrm{CF}$ 412 CNC). The workpiece material was HSS - high speed steel (B.S. BM 42) at 66 HRc hardness. The tool was aluminum oxide wheel ( $\gg$ Winterthur $\ll$ type 53 A80 F15V PMF, diameter $\boldsymbol{D}_{s}=$ 

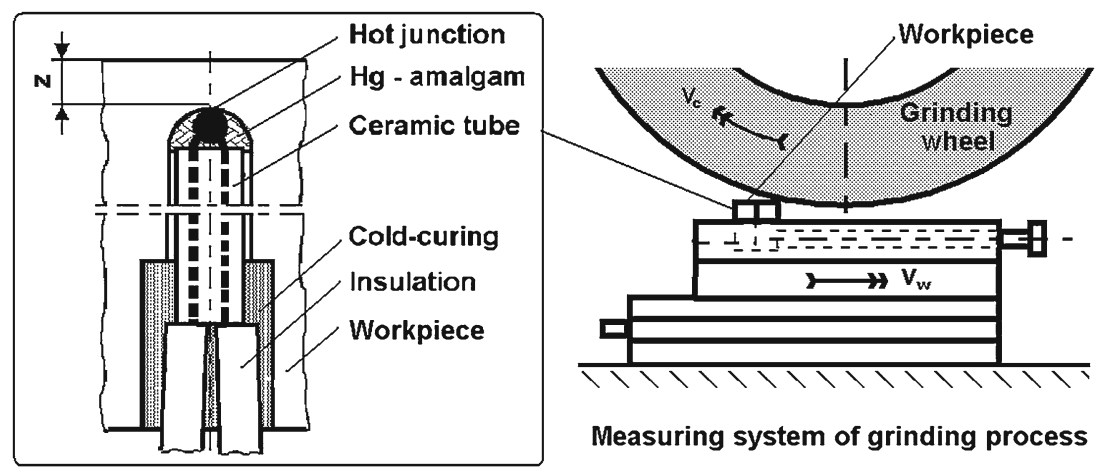

Measuring system of grinding process

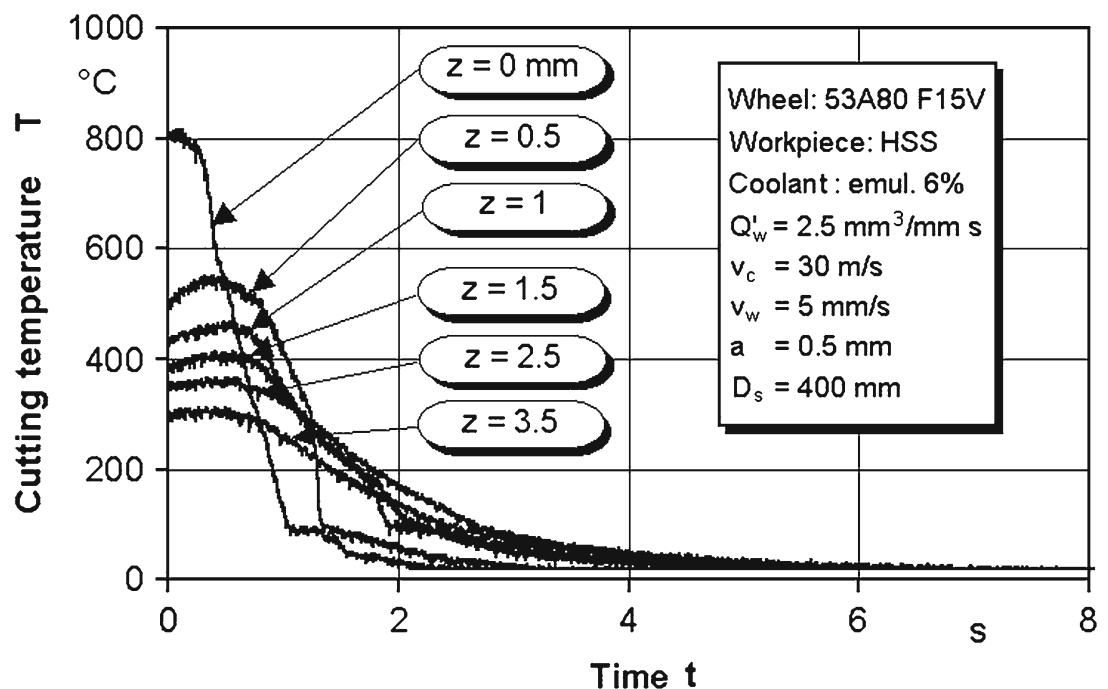

Figure 5. Experimentally obtained temperature distribution in time within the workpiece surface layer.

$400 \mathrm{~mm}$ ). The depth of cut was $\boldsymbol{a}=0,5 \mathrm{~mm}$, the workpiece speed was $\boldsymbol{v}_{\boldsymbol{w}}=5 \mathrm{~mm} / \mathrm{s}$ and the wheel speed was $\boldsymbol{v}_{s}=30 \mathrm{~m} / \mathrm{s}$. A water-based coolant (emulsion 6\%) was used during the machining test.

For measurement, processing and control of cutting temperatures, a modern information system was used. The temperature was measured in the workpiece surface layer using a thermocouple (type $\mathrm{K}, \phi 0,2 \mathrm{~mm}$ ) built into the workpiece at a specified clearance from the tool/ workpiece interface, figure 5(top). Application of thermocouple is simple, reliable and costefficient, and does not interfere with the real machining conditions.

\subsection{The results of inverse method}

In this case of verification, to investigate the machining thermal process by inverse heat transfer problem, following input parameters were taken. Thermo-physical properties of workpiece material (high speed steel B.S. BM 42): thermal conductivity $\boldsymbol{k}=21,378+0,0275 \cdot \boldsymbol{T} \mathrm{W} / \mathrm{m}^{\circ} \mathrm{C}$ and thermal diffusivity $\alpha=7.5 \cdot 10^{-6} \mathrm{~m}^{2} / \mathrm{s}$. Spatial discretization: number of the interior points $\boldsymbol{h}=7$ with space step $\Delta \boldsymbol{x}=0,5 \mathrm{~mm}$. Temporal discretization: number of the time increment 
$\boldsymbol{m}=25$ with time step $\Delta \boldsymbol{t}=0,025 \mathrm{~s}$. Initial temperature distribution: experimentally determined temperatures (figure 5) for the $t=0 \mathrm{~s}$. Boundary condition: heat flux over the lower boundary of the considered workpiece surface layer $\boldsymbol{q}^{*}(t)=0 \mathrm{~W} / \mathrm{m}^{2}$. Additional condition: the known temperature distribution measured outside the workpiece at depth $z=1 \mathrm{~mm}$ (figure 5), i.e., at point $\boldsymbol{K}=2$.

Based on the previously presented, the total temperature field in workpiece first was obtained by computation. Calculated temperature change over time in the workpiece surface layer, as well as the model the temperature field in the cutting zone, are shown in figure 6 . The temperature distribution in the workpiece is computed by inverse heat transfer problem. It defines the thermal process in the machining.

The computed time and depth-related change of temperature in the interface zone of the workpiece surface layer (figure 6), shows a high degree of conformity with the experimentally obtained results (figure 5).

Once temperature field in the workpiece is determined, the unknown heat flux in the tool/workpiece interface is calculated. In this case of verification, the contact temperature was not allowed to exceed the critical tempering temperature, which was experimentally established at $550^{\circ} \mathrm{C}$ for the selected high speed steel. The computed temperature and heat flux, shown in figure 7, define the heat loading in the workpiece surface layer. The computed distribution of heat flux over the tool/workpiece interface clearly shows the direct relationship between heat flux parameters, i.e., the power of heat and its total active time. The greater the power of heat flux, the shorter the active time, and vice versa.

\subsection{Optimization of heat flux parameters}

Using extreme case of inverse heat transfer problem for optimization of the machining thermal process requires selection of machining conditions such that the surface heat loading does not exceed the limits allowed. Thus, optimization of heat loading of workpiece surface layer is required, which relies on heat flux parameters. More specifically, the process of optimization

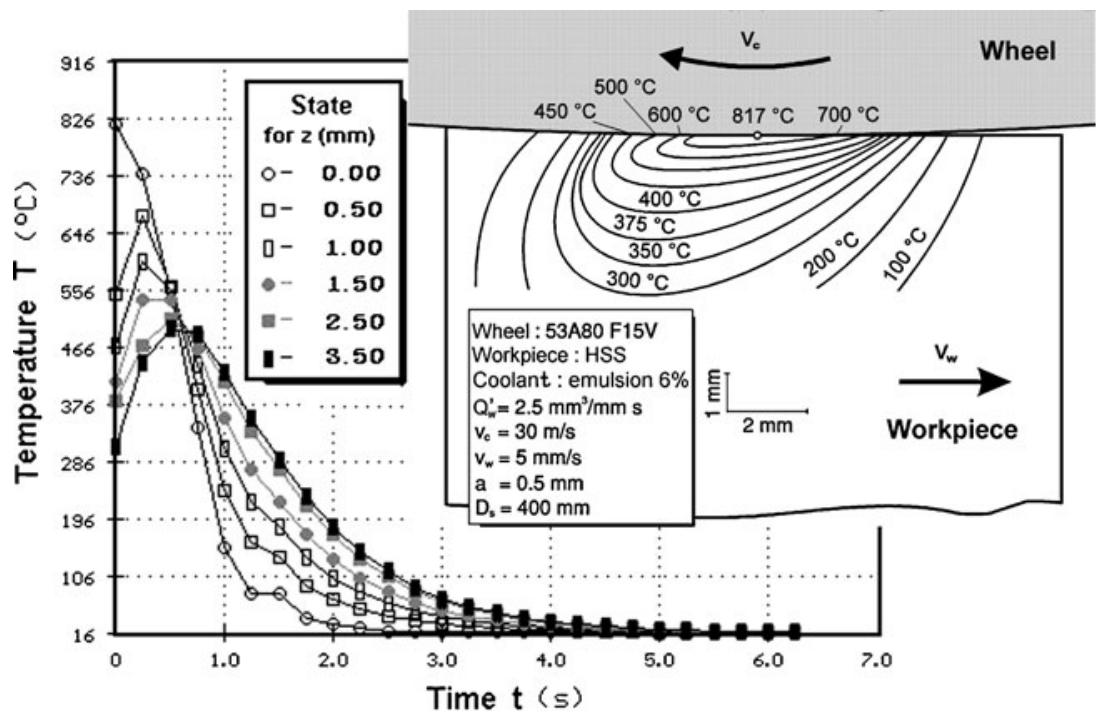

Figure 6. Computation of temperature field in the workpiece surface layer. 
means determination of most favourable ratio between heat power and its active time, for the previously established distribution of heat flux over the tool/workpiece interface, figure 7 .

Under the condition of maximum machining productivity $\left(Q_{w}^{\prime}=v_{w} \cdot a=\max \right)$, the process of optimization is conducted by completely searching the bounded solution space $\left(v_{w}, a\right)$. Thus optimal workpiece speed $\boldsymbol{v}_{w}$ and cutting depth $\boldsymbol{a}$ are determined which can be inside or on the very boundary of the search space.

Based on the previously proposed model of optimization, the defined criterion of optimization and the state functions and boundaries, the optimal ratio of heat flux parameters is calculated and subsequently used to derive optimal machining conditions, figure 8. Parameters thus derived,

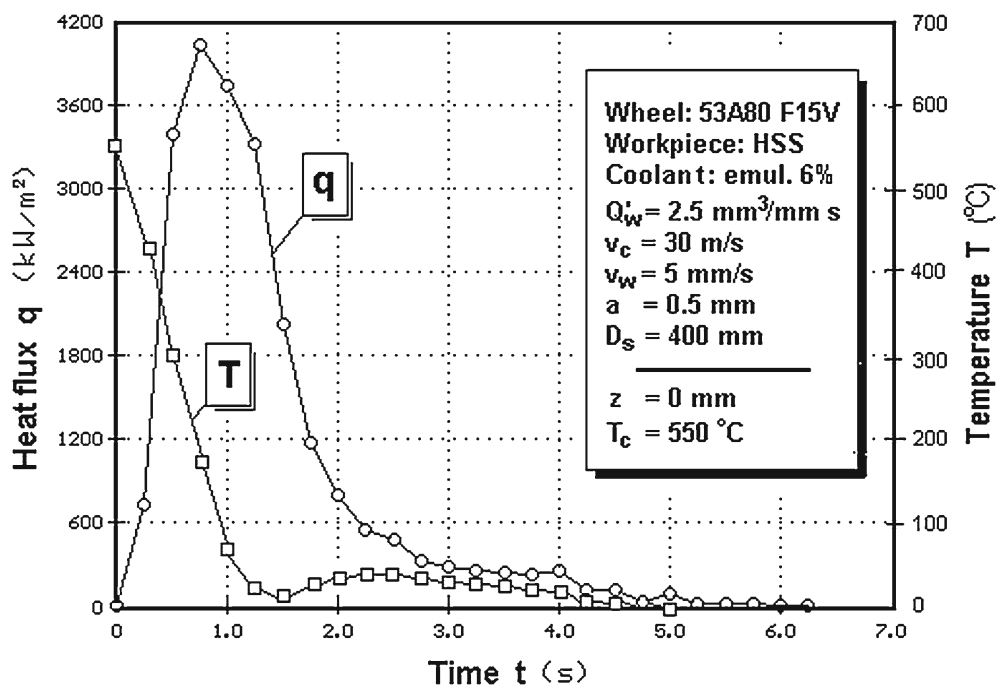

Figure 7. Heat flux and temperature distribution over the tool/workpiece interface.

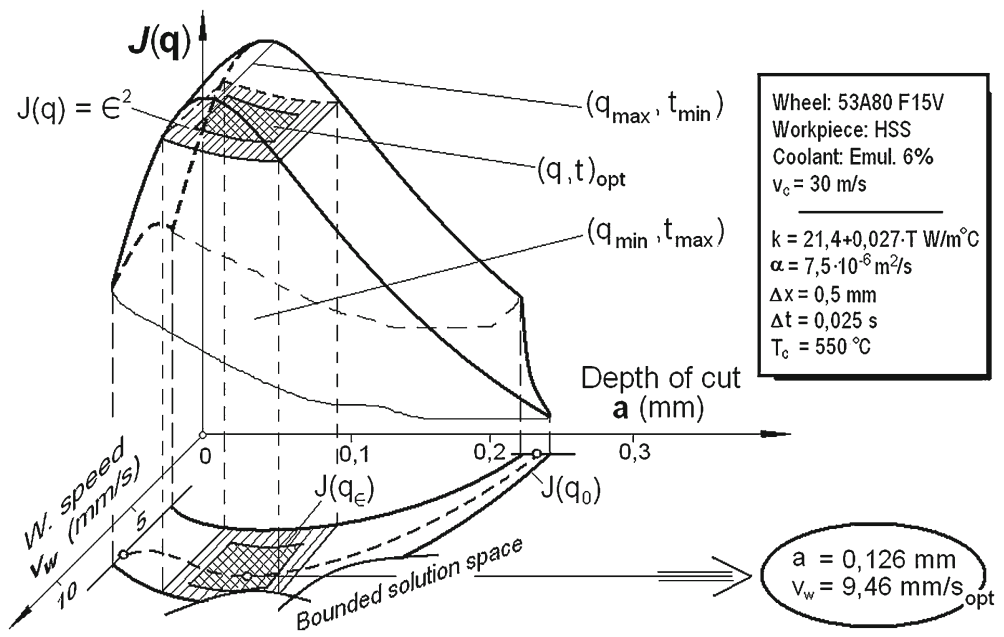

Figure 8. Optimal machining conditions is calculated of most favourable ratio between heat power $\boldsymbol{q}$ and its active time $t$. 
yield maximum machining productivity with the temperature being kept below the dangerous level which could compromise functional properties of finished parts.

The comparison between the computed optimal machining conditions with the maximum allowed ones, which were derived experimentally, shows very little differences. The differences can be explained by a large number of parameters which were either omitted from analysis or had to be estimated in the course of analytical modelling of the machining process.

\section{Conclusions}

Based on this investigation, following conclusions can be made:

- The inverse heat transfer problems are applied in identification, design, control and optimization of machining thermal processes based on analytical models and experimental results is gaining popularity.

- In the optimization over thermal state in machining, the extreme case of inverse heat transfer problem is practically the only way to reliably approximate the allowed heat loading on workpiece and tool.

- Analytical inverse heat transfer problem allows approximation of a complete temperature field and heat flux distribution in the cutting zone.

- The inverse heat transfer problem was solved using method of finite differences in implicit form, where the concept of this method very much resembles the physical process.

- As this is an experimental and analytical system for computation of heat loading of machining process, it requires an exact, experimentally obtained temperature distribution at a single point within the cutting zone.

- The stability of the inverse problem numerical solution largely depends on the initial and boundary conditions, thermal and physical properties of the machining process and the choice of temporal and spatial discretization.

- The analytically obtained temperature field in the cutting zone largely agrees with the experimental results.

- Optimization of thermal state of cutting zone requires determination of the function of the process state and the control function, so as to satisfy the analytical form of the inverse heat transfer problem.

- Total search iterative method was selected as the method of optimization.

- Optimization of machining conditions allows the parameters of heat flux to be kept within limits which guarantee functional properties of the tool and workpiece.

\section{List of symbols}

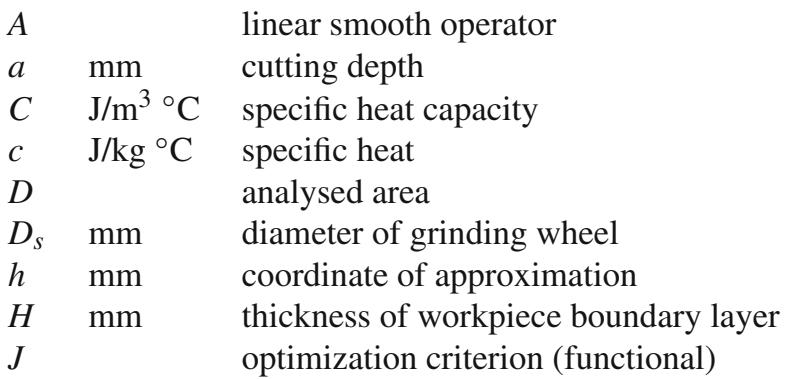




\begin{tabular}{|c|c|c|}
\hline$K$ & & point outside of workpiece \\
\hline$k$ & $\mathrm{~W} / \mathrm{m}{ }^{\circ} \mathrm{C}$ & thermal conductivity \\
\hline$Q_{w}^{\prime}$ & $\mathrm{mm}^{3} / \mathrm{mm} \mathrm{s}$ & specific material removal rate \\
\hline$q$ & $\mathrm{~W} / \mathrm{m}^{2}$ & heat flux \\
\hline$q^{*}$ & $\mathrm{~W} / \mathrm{m}^{2}$ & known heat flux \\
\hline$n$ & & time of approximation \\
\hline$r_{c}$ & & weight coefficient factor \\
\hline$T$ & ${ }^{\circ} \mathrm{C}$ & temperature \\
\hline$T_{c}$ & ${ }^{\circ} \mathrm{C}$ & critical tempering temperature \\
\hline$T_{0}$ & ${ }^{\circ} \mathrm{C}$ & initial temperature \\
\hline$T_{K}$ & ${ }^{\circ} \mathrm{C}$ & known temperature at point $K$ \\
\hline$T_{q}$ & ${ }^{\circ} \mathrm{C}$ & calculated temperature \\
\hline$t$ & $\mathrm{~s}$ & time \\
\hline$t_{m}$ & $\mathrm{~s}$ & largest time increment \\
\hline$v_{c}$ & $\mathrm{~m} / \mathrm{s}$ & cutting speed \\
\hline$v_{w}$ & $\mathrm{~mm} / \mathrm{s}$ & workpiece speed \\
\hline$u$ & & input parameter \\
\hline$x$ & $\mathrm{~mm}$ & coordinate \\
\hline$z$ & & output parameter \\
\hline$z$ & $\mathrm{~mm}$ & distance of from machined surface \\
\hline$\alpha$ & $\mathrm{m}^{2} / \mathrm{s}$ & thermal diffusivity \\
\hline$\Delta t$ & s & time increment \\
\hline$\Delta x$ & $\mathrm{~mm}$ & elementary piece \\
\hline$\varepsilon$ & & error \\
\hline$\rho$ & $\mathrm{kg} / \mathrm{m}^{3}$ & material density \\
\hline$\Omega$ & & stabilizing functional \\
\hline
\end{tabular}

\section{References}

Alifanov O M 1994 Inverse heat transfer problems. (Berlin: Springer-Verlag)

Andreas N A 1989 An inverse finite element method for directly formulated free boundary problems. Int. J. Numerical Methods in Eng., 28: 2383-2396

Beck J V, Blackwell B, Clair C R 1985 Inverse Heat Conduction: Ill-posed Problems. (New York: WileyIntercience Publication)

Gostimirovic M, Kovac P 2008 The thermal state of the workpiece surface layer during productivity grinding. Manufacturing Technology Journal for Science, Research and Production, Prague, 8: 55-61

Kim H J, Kim N K, Kwak J S 2006 Heat flux distribution model by sequential algorithm of inverse heat transfer determining workpiece temperature in creep feed grinding. International Journal of Machine Tools and Manufacture, No 46: 2086-2093

Khachfe R A, Jarny Y 2000 Numerical solution of 2-D non linear inverse heat conduction problems using finite element techniques. Numerical Heat transfer, An International Journal of Computation and Methodology, Part B, 37: 45-68

Kreith F, Black W 1980 Basic heat transfer. (New York: Harper and Row)

Lin J 1995 Inverse estimation of the tool-work interface temperature in end milling. International Journal of Machine Tools and Manufacture, 35(5): 751-760

Milikic D, Gostimirovic M 1994 Influence of tribological and geometrical parameters of the grinding process on the magnitude of cutting temperature. Tribologija u industriji, 16(3): 77-84

Özisik N M, Orlande R B 2000 Inverse heat transfer: fundamental and applications. (New York: Taylor \& Francis) 
Shaw M C 2003 The size effect in metal cutting. Sadhana, 28(Part 5): 875-896

Shidfor A, Tawakoli K 2002 An inverse heat conduction problem. Southeast Asion Bulletion of Mathematics, 26: 503-507

Stephenson D A 1991 An inverse method for investigating deformation zone temperatures in metal cutting. Journal of Engineering for Industry, 113: 129-136

Tikhe A K, Deshmukh K C 2005 Inverse transient thermoelastic deformations in thin circular plates. Sadhana, 30(Part 5): 661-671 